\title{
Examination of Quaternary
} Ammonium Compound Resistance in Proteus mirabilis Isolated from Cooked Meat Products in China

\section{OPEN ACCESS}

Edited by: Avelino Alvarez-Ordóñez, University of León, Spain

Reviewed by:

Beatrix Stessl,

Veterinärmedizinische Universität

Wien, Austria

Dara Leong,

Teagasc, The Irish Agriculture and Food Development Authority, Ireland

${ }^{*}$ Correspondence: Tao Yu

yutao7777@hotmail.com

Specialty section: This article was submitted to

Food Microbiology, a section of the journa

Frontiers in Microbiology

Received: 28 June 2017 Accepted: 22 November 2017 Published: 11 December 2017

Citation:

Jiang X, Yu T, Liu L, Li Y, Zhang K, Wang $H$ and Shi $L$ (2017)

Examination of Quaternary Ammonium Compound Resistance in Proteus mirabilis Isolated from Cooked Meat Products in China.

Front. Microbiol. 8:2417. doi: 10.3389/fmicb.2017.02417

\author{
Xiaobing Jiang ${ }^{1}$, Tao Yu ${ }^{2 *}$, Lei Liu ${ }^{1}$, Yi Li ${ }^{1}$, Kun Zhang ${ }^{1}$, Hailei Wang ${ }^{1}$ and Lei Shi ${ }^{3}$ \\ ${ }^{1}$ College of Life Sciences, Henan Normal University, Xinxiang, China, ${ }^{2}$ College of Life Science and Technology, \\ Xinxiang University, Xinxiang, China, ${ }^{3}$ Institute of Food Safety and Nutrition, Jinan University, Guangzhou, China
}

The aim of this study was to examine the presence of genes responsible for resistance to quaternary ammonium compounds (QACs) and the association of qac genes with class 1 integrons in Proteus mirabilis isolated from cooked meat products. A total of 52 P. mirabilis isolates (29.2\%) were detected from 178 samples, and their minimum inhibitory concentrations (MICs) of benzalkonium chloride (BC) ranged from 4 to $>32 \mu \mathrm{g} / \mathrm{mL}$. The isolates with BC MICs of $24 \mu \mathrm{g} / \mathrm{mL}$ were observed most frequently. PCR assays indicated that $m d f A, y d g E / y d g F$, qacE, qacE $\Delta 1$, emrE, sugE(c), and $\operatorname{sug} E(p)$ were commonly present $(32.7 \%-100 \%)$ in these isolates, but qacH was less prevalent (3.8\%). Five groups of resistance gene cassettes were identified in 10 int/1-positive isolates. An unusual gene cassette array dfrA32-ereA-aadA2 was found in one foodborne isolate of $P$. mirabilis. Two isolates harbored $q a c H$ - and sul3- associated non-classic integrons: aadA2-cmIA1-aadA1-qacH-IS440-sul3 and a new arrangement dfrA32-ereA1-aadA2-cmIA1-aadA1-qacH-IS440-sul3, which is first reported in $P$. mirabilis. Non-classic class 1 integrons were located on conjugative plasmids of $100 \mathrm{~kb}$ in two tested isolates. Our data showed that the QAC resistance genes were commonly present among $P$. mirabilis isolates from cooked meats and qacH was associated with non-classic class 1 integrons. The creation of transconjugants demonstrated that qacH-associated non-classic class 1 integrons were located on conjugative plasmids and therefore could facilitate the co-dissemination of disinfectant and antimicrobial resistance genes among bacteria, an increasing area of concern.

Keywords: Proteus mirabilis, qac genes, integron, benzalkonium chloride, resistance

\section{INTRODUCTION}

Proteus mirabilis, widely distributed in the natural environment, is a member of Enterobacteriaceae family. As an opportunistic pathogen, $P$. mirabilis can cause urinary tract and wound infections. In addition, it can also contaminate meat (Kim et al., 2005; Wong et al., 2013), vegetables (Uzeh et al., 2009), and seafood (González-Rodríguez et al., 2002), and has been associated with food poisoning (Wang et al., 2010). Previous studies focused on the antimicrobial resistance and the distribution of resistance genes among foodborne P. mirabilis (Kim et al., 2005; Wong et al., 2013). Unlike other foodborne bacteria (Zou et al., 2014; Zhang et al., 2016), little data have been reported 
on the disinfectant resistance and molecular mechanisms underlying resistance in foodborne $P$. mirabilis. Disinfectants have the ability to co-select for antimicrobial resistance when these traits are genetically linked (Chapman, 2003). Moreover, much evidence has shown that disinfectant and antimicrobial resistance genes can be co-transferred between bacteria via horizontal gene transfer (Zhao et al., 2009; Call et al., 2010; Sáenz et al., 2010; Partridge et al., 2012), which poses a risk to public health.

Benzalkonium chloride (BC), an important representative of quaternary ammonium compounds (QACs), is used extensively as a disinfectant in the food processing environment to prevent the growth of microorganisms and to ensure the microbiological safety of food products. Its widespread use, however, may impose a selective pressure for resistant strains of bacteria (Cruz and Fletcher, 2012). In the past decades, resistance to BC (resistant breakpoints for BC were $>30 \mu \mathrm{g} / \mathrm{mL}$ in Sidhu et al. (2002) and $\geq 20 \mu \mathrm{g} / \mathrm{mL}$ in Meier et al. (2017)) has been reported in many bacterial isolates from food and food processing plants (Sidhu et al., 2002; Meier et al., 2017).

Efflux pumps, QacE, QacE $\Delta 1$, QacF, QacG, QacH/I, and $\operatorname{SugE}(\mathrm{p})$, which contribute for $\mathrm{BC}$ resistance, have been identified in Gram-negative bacteria (Zou et al., 2014). They are members of the small multidrug resistance (SMR) family and are generally located on mobile genetic elements, such as integrons and plasmids (Zou et al., 2014). qac are closely associated with class 1 integrons. qacE is located in the $3^{\prime}$-conserved segment (CS) of class 1 integrons and qacE $\Delta 1$, a deletion derivative of qacE, confers increased resistance to BC (Kazama et al., 1998). qacF shows a high degree of similarity (67.8\% identity) with qacE. Class 1 integrons carrying qacG have been found in Gram-negative bacteria (Chu et al., 2001; Partridge et al., 2012). qacH, which was first identified in Staphylococcus (Heir et al., 1998), has been observed frequently in Gram-negative bacteria (Hegstad et al., 2010) and it confers a broader resistance phenotype compared with qacG (Heir et al., 1998). qacH is usually recognized as an important component of class 1 integron that lacks the normal $3^{\prime}$-CS region (i.e., a "nonclassic class 1 integron"), which has been detected in many species of Enterobacteriaceae (Antunes et al., 2007; Chang et al., 2009, 2011; Sáenz et al., 2010; Farkas et al., 2016). Several studies found $q a c H$ gene and $\beta$-lactamase genes (bla $a_{\mathrm{IMP}-15}$, $b l a_{G E S-1}, b l a_{G E S-5}$, and bla $a_{\mathrm{OXA}-2}$ ) linked to class 1 integrons from Pseudomonas aeruginosa clinical isolates (Garza-Ramos et al., 2008, 2010; Viedma et al., 2009). qacH-aadB has been reported from environmental bacteria, including Paracoccus versutus, Brevundimonas diminuta, Brachymonas denitrificans, Stenotrophomonas acidaminiphila, and Psychrobacter spp. (Li et al., 2009) and qacH-aadA8 from Vibrio cholerae (Ceccarelli et al., 2006). Additionally, qacH located on a novel transposon Tn6188 was found in Listeria monocytogenes (Müller et al., 2013). In several studies, $q a c H$ in Enterobacteriaceae was renamed as qacI to distinguish it from qacH from Staphylococcus (Naas et al., 2001; Curiao et al., 2011). For this paper, we still use the gene name "qacH". qacH exhibits $91.6 \%$ similarity with the sequence of $q a c F$. Finally, $\operatorname{sugE}(p)$ is frequently present on multidrug resistance plasmids, that have been reported in
Escherichia coli and Salmonella (Zhao et al., 2009; Call et al., 2010).

Five chromosome-encoded efflux pump genes (sugE(c), emrE, $m d f A$, and $y d g E / y d g F)$ have been reported to confer resistance to BC (Bay and Turner, 2009; Zou et al., 2014). In addition to $m d f A$ encoding a multidrug resistance efflux pump belonging to the major facilitator superfamily (MFS), the remaining genes encode the SMR family efflux pumps.

The aims of this study were to assess the $\mathrm{BC}$ resistance and investigate the presence of disinfectant resistance genes and the association of qac genes with class 1 integrons among the $P$. mirabilis isolates from cooked meat products in China.

\section{MATERIALS AND METHODS}

\section{P. mirabilis Isolates}

Between January and September 2015, 178 samples (250 g) of cooked meat products, including roasted meats $(n=67)$ and sauced meats $(n=111)$, were purchased from supermarkets and cooked meat shops in Xinxiang, a city of Henan. Six local supermarkets and ten cooked meat shops were randomly selected and each site was visited once. Thirteen samples were collected from each supermarket and ten samples from each cooked meat shops. Samples were transported to the laboratory in an icebox and were processed immediately for bacterial isolation. According to the previous study, the traditional Salmonella isolation protocol was modified to isolate P. mirabilis (Wong et al., 2013). Briefly, a rinse was performed by adding $25 \mathrm{~g}$ of sample to $225 \mathrm{~mL}$ of buffer peptone water (BPW; Huankai Ltd., Guangzhou, Guangdong, China) in sterile lateral filter bags with thorough mixing by using a homogenizer (BagMixer lab blender 400; Interscience, SaintNom-La-Breteche, France). These samples were then incubated at $37^{\circ} \mathrm{C}$ for $18 \mathrm{~h}$. Pre-enriched sample $(1 \mathrm{~mL})$ was inoculated into $10 \mathrm{~mL}$ of tetrathionate broth base (TTB; Huankai) and incubated at $42^{\circ} \mathrm{C}$ for $24 \mathrm{~h}$. A loop of inoculum was streaked onto xylose lysine deoxycholate agar (XLD; Huankai) and incubated for $24 \mathrm{~h}$ at $37^{\circ} \mathrm{C}$. Three to four Salmonella-like colonies (pink with or without black center on XLD; and yellow with or without black center colonies on XLD were also considered as suspected colonies) were picked and re-streaked on nutrient agar (NA). The isolates with swarming phenotype were identified by using the API 20E bacterial identification system (BioMerieux, Marcy l'Etoile, France). All isolates designated as P. mirabilis were additionally analyzed by PCR-based $16 \mathrm{~S}$ rDNA sequencing using a pair of universal primers 27F/1492R (Table 1). Only one isolate from each sample was selected for further characterization.

\section{Determination of Minimum Inhibitory Concentrations (MICs) for BC}

The MICs of BC for P. mirabilis were determined using the agar dilution method recommended by the Clinical and Laboratory Standards Institute (Clinical and Laboratory Standards Institute, 2012). BC was tested in concentration range of $4-32 \mu \mathrm{g} / \mathrm{mL}$. 
TABLE 1 | Primers used in this study.

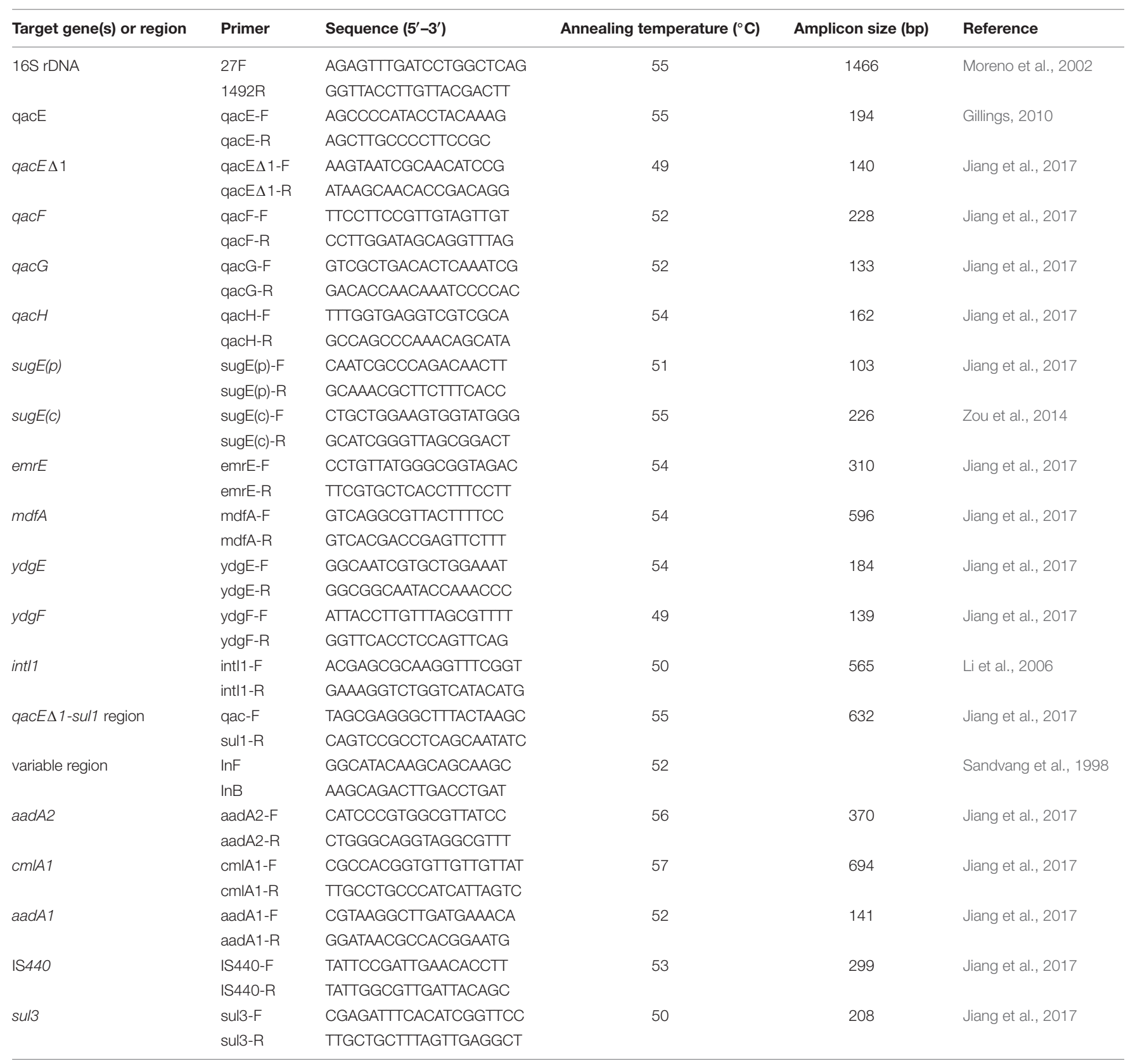

$P$. mirabilis suspensions were adjusted to a turbidity equivalent to that of a $0.5 \mathrm{McF}$ arland standard with sterilized saline solution (0.9\%) and delivered to the Mueller-Hinton (MH; Huankai) agar containing different concentrations of BC (Aladdin Biochemical Technology Co., Ltd., Shanghai, China). The plates were incubated at $37^{\circ} \mathrm{C}$ for $24 \mathrm{~h}$. The lowest concentration of $\mathrm{BC}$ that prevented growth was considered as the MIC. Each of the tests was done in triplicate. In cases in which not all three replicates had the same results, the MICs were determined once more. Escherichia coli ATCC 10536 (a gift from Lijun Zhou, Navy General Hospital, Beijing, China) was used as a quality control strain (the MIC of this strain for BC was $16 \mu \mathrm{g} / \mathrm{mL})$.

\section{Detection of QAC Resistance Genes}

All isolates were screened by PCR for the presence of qac genes, including qacE, qacE $\Delta 1, q a c F, q a c G, q a c H, \operatorname{sug} E(p), \operatorname{sug} E(c)$, emrE, $m d f A$, and $y d g E / y d g F$ (Table 1). Colonies were transferred to an Eppendorf tube filled with water and boiled to prepared DNA template (Wang et al., 2008). The PCR mixture consisted of $2.5 \mu \mathrm{L}$ of boiled lysate DNA, $0.6 \mu \mathrm{M}$ (each) primer, $200 \mu \mathrm{M}$ deoxynucleoside triphosphate (Takara Bio Inc., Otsu, Shiga, Japan), 1× PCR buffer (Takara), 0.5 U Taq DNA polymerase (Takara) in a total volume of $25 \mu \mathrm{L}$. The PCR conditions were as follows: initial denaturation at $94^{\circ} \mathrm{C}$ for $5 \mathrm{~min}$ followed by 30 cycles of denaturation at $94^{\circ} \mathrm{C}$ for $30 \mathrm{~s}$, annealing at different temperatures (between 49 and $55^{\circ} \mathrm{C}$ depending on the primer set) 
for $30 \mathrm{~s}$, extension at $72^{\circ} \mathrm{C}$ for $30 \mathrm{~s}$, and a final extension at $72^{\circ} \mathrm{C}$ for $7 \mathrm{~min}$. The purified PCR products were sequenced and DNA sequence data were analyzed using the BLAST program ${ }^{1}$.

\section{Characterization of Class 1 Integrons}

All isolates were screened for intI1 (Table 1). Because the occurrence of sulfonamide resistance gene (sul1) and quaternary ammonium compounds resistance gene $(q a c E \Delta 1)$ is often associated with classic class 1 integrons, presence of qacE $\Delta 1$-sull region in all intI1-positive isolates was tested by using the primers qac-F and sul1-R (Table 1). The presence of gene cassettes in variable regions were characterized by PCR using specific primers (Table 1). Primers InF and InB were used for classic class 1 gene cassettes amplifications; and primers InF and aadA2-R were used for non-classic class 1 integron amplifications. To determine the genetic structure of non-classic class 1 integrons, a wide variety primers were designed. PCR “primer-walking” strategy was used to amplify overlapped individual fragments using Takara LA Taq DNA polymerase to get the complete arrangement. All the obtained amplicons were sequenced on both strands.

\section{Genetic Locations of qacH-Associated Class 1 Integrons}

Plasmid DNA was isolated from two qacH-positive isolates using TIANpure Mini Plasmid Kit (TIANGEN Biotech Co., Ltd., Beijing, China). To determine the number and size of plasmids, genomic DNA from qacH-positive isolates was performed by S1 nuclease (Takara) digestion prior to PFGE. S1PFGE fragments were transferred onto membranes (Amersham Pharmacia Biotech, Buckinghamshire, United Kingdom) by Southern blot and hybridized with specific probes of intI1 and qacH genes. The probes were made with the DIG-High Prime DNA Labeling and Detection Starter Kit I (Roche Applied Science, Mannheim, Germany).

\section{Conjugation Experiments}

The transfer of plasmids carrying qacH-associated class 1 integrons was studied by performing conjugation experiments as described previously (Koo and Woo, 2011). E. coli J53 Az (a sodium azide resistant strain) was used as the recipient and isolates containing non-classic class 1 integron severed as donors. Briefly, donor and recipient cells were mixed with each other at $1: 10$ ratio and incubated at $37^{\circ} \mathrm{C}$ overnight. Transconjugants were selected on trypticase soy agar (TSA; Huankai) plates containing sodium azide $(150 \mu \mathrm{g} / \mathrm{mL}$; Sinopharm Chemical Reagent Co., Ltd., Shanghai, China), streptomycin (50 $\mu \mathrm{g} / \mathrm{mL}$; Sigma-Aldrich, St. Louis, MO, United States) and chloramphenicol (16 $\mu \mathrm{g} / \mathrm{mL}$; SigmaAldrich). PCR was used to confirm that the transconjugants carried the same resistance genes as their donors.

\section{Statistical Analysis}

The statistical package SPSS 15.0 (SPSS Inc., Chicago, IL, United States) was used, and the two-tailed paired Student's

${ }^{1}$ http://www.ncbi.nlm.nih.gov/BLAST/ $t$-test was applied to determine the significance of differences. A $P$-value $<0.05$ was considered statistically significant for comparisons.

\section{Nucleotide Sequence Accession Numbers}

The nucleotide sequences of the qacH-carrying integrons have been submitted to GenBank under accession numbers KY662007 for aadA2-cmlA1-aadA1-qacH-IS440-sul3 and KY426918 for dfrA32-ereA1-aadA2-cmlA1-aadA1-qacH-IS440-sul3.

\section{RESULTS}

\section{Isolation of $\boldsymbol{P}$ mirabilis}

Colony characteristics of $P$. mirabilis on XLD agar were similar to those of Salmonella. In this study, Salmonella-like colonies were found in 88 samples (Supplementary Table S1). Among these samples, fifty-six samples were positive for isolates with swarming phenotype (Supplementary Table S1). These suspected $P$. mirabilis isolates were identified by the biochemical tests and PCR-based 16S rDNA sequencing. Fifty-two samples were positive for $P$. mirabilis, five samples were positive for $P$. vulgaris, and one sample was positive for Salmonella (Supplementary Table S1).

\section{Susceptibility of $P$. mirabilis Isolates to BC}

A total of $52(29.2 \%)$ P. mirabilis isolates were recovered from 178 cooked meat samples (Supplementary Table S1). For these isolates, the MICs of BC ranged from 4 to $>32 \mu \mathrm{g} / \mathrm{mL}$ (Figure 1 and Supplementary Table S2). The isolates with BC MICs of $24 \mu \mathrm{g} / \mathrm{mL}$ ( $n=17$, accounting for $32.7 \%$ of all isolates) were

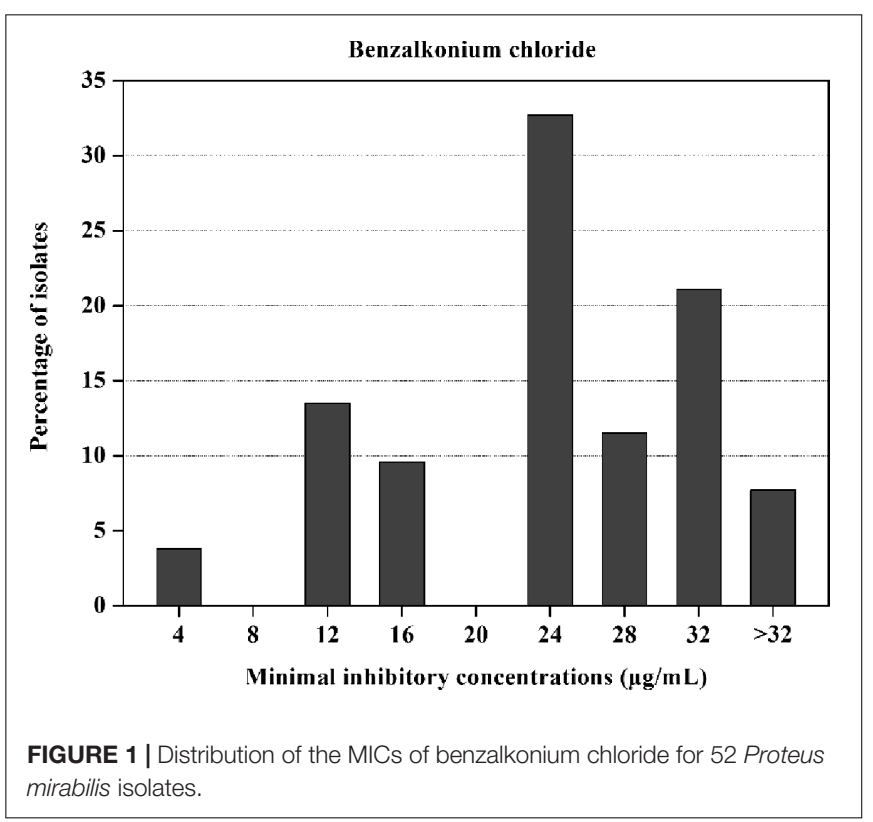


TABLE 2 | Different resistance gene combinations in Proteus mirabilis isolates.

\begin{tabular}{|c|c|c|c|c|c|c|c|c|}
\hline \multirow[t]{2}{*}{ Gene combination } & \multicolumn{7}{|c|}{ Number of isolates with MIC $(\mu \mathrm{g} / \mathrm{mL})$ as follows } & \multirow[t]{2}{*}{ Total } \\
\hline & 4 & 12 & 16 & 24 & 28 & 32 & $>32$ & \\
\hline qacE-qacE $\Delta 1-m d f A-y d g E / y d g F$ & & 4 & 1 & 9 & & & & 14 \\
\hline$m d f A-y d g E / y d g F$ & 2 & 3 & 2 & & & & & 7 \\
\hline sugE(p)-qacE-qacE $\Delta 1$-emrE-mdfA-ydgE/ydgF & & & & 1 & 1 & 4 & & 6 \\
\hline $\operatorname{sugE}(c)-s u g E(p)-q a c E-q a c E \Delta 1-e m r E-m d f A-y d g E / y d g F$ & & & & & & 2 & 2 & 4 \\
\hline $\operatorname{sugE}(c)$-qacE-qacE $\Delta 1$-emrE-mdfA-ydgE/ydgF & & & & 3 & 1 & & & 4 \\
\hline emrE-mdfA-ydgE/ydgF & & & 2 & 1 & 1 & & & 4 \\
\hline $\operatorname{sug} E(c)-s u g E(p)-m d f A$ & & & & & 1 & 3 & & 4 \\
\hline $\operatorname{sug} E(c)$-emrE-mdfA-ydgE/ydgF & & & & 2 & 1 & & & 3 \\
\hline $\operatorname{sug} E(c)-s u g E(p)-e m r E-m d f A-y d g E / y d g F$ & & & & & & 2 & & 2 \\
\hline$s u g E(c)-m d f A-y d g E / y d g F$ & & & & 1 & 1 & & & 2 \\
\hline $\operatorname{sug} E(c)-q a c H-m d f A-y d g E / y d g F$ & & & & & & & 1 & 1 \\
\hline $\operatorname{sug} E(c)-s u g E(p)-q a c H-m d f A$ & & & & & & & 1 & 1 \\
\hline
\end{tabular}

observed most frequently, followed by the isolates with MICs of $32 \mu \mathrm{g} / \mathrm{mL}(n=11,21.1 \%)$.

\section{Presence of QAC Resistance Genes}

The presence of QAC resistance genes among $P$. mirabilis isolates are presented in Supplementary Table S2. The $m d f A$ gene was the most widespread QAC resistance gene, being found in $100 \%$ of isolates, followed by $y d g E / y d g F(90.4 \%, n=47)$, qacE $(53.8 \%$, $n=28), q a c E \Delta 1(53.8 \%, n=28), \operatorname{emrE}(44.2 \%, n=23), \operatorname{sugE}(c)$ $(40.4 \%, n=21), \operatorname{sugE}(p)(32.7 \%, n=17)$, and $q a c H(3.8 \%$, $n=2)$. The $q a c F$ and $q a c G$ genes were not detected in any of the isolates. The top two QAC resistance genotypes were qacE$q a c E \Delta 1-m d f A-y d g E / y d g F(26.9 \%)$ and $m d f A-y d g E / y d g F(13.5 \%)$ (Table 2).

Our results showed that $q a c E$ always occurred simultaneously with qacE $\Delta 1$, but the MICs of BC were not significantly different $(P>0.05)$ between qacE-qacE $\Delta 1$-positive and -negative isolates. The presence of $\operatorname{sug} E(p)$ was significantly associated with the higher MICs of BC $(P<0.05)$. Among the $17 \operatorname{sugE}(p)$-positive isolates, $82.4 \%(n=14)$ had the MICs of $\geq 32 \mu \mathrm{g} / \mathrm{mL}$. High MICs of BC $(>32 \mu \mathrm{g} / \mathrm{mL})$ were also observed in the two isolates that carried qacH.

\section{Genetic Structure of Class 1 Integrons}

The 52 P. mirabilis isolates were subjected to the PCR screening for the expected integrase gene, and the 565-bp corresponding amplicon was detected in 10 isolates, consistent with the presence of the class 1 integrase gene. Five groups of resistance gene cassettes, named as type I-V, were identified in these isolates (Figure 2), including: $d f r A 17-a a d A 5, d f r A 5, d f r A 1-o r f C, d f r A 32-$ ereA1-aadA2, and aadA2 (Table 3).

\section{Analysis of Plasmids Carrying qacH-Associated Class 1 Integrons}

According to the bands obtained by S1-PFGE of qacH-positive isolates, the number and size of their plasmids were determined

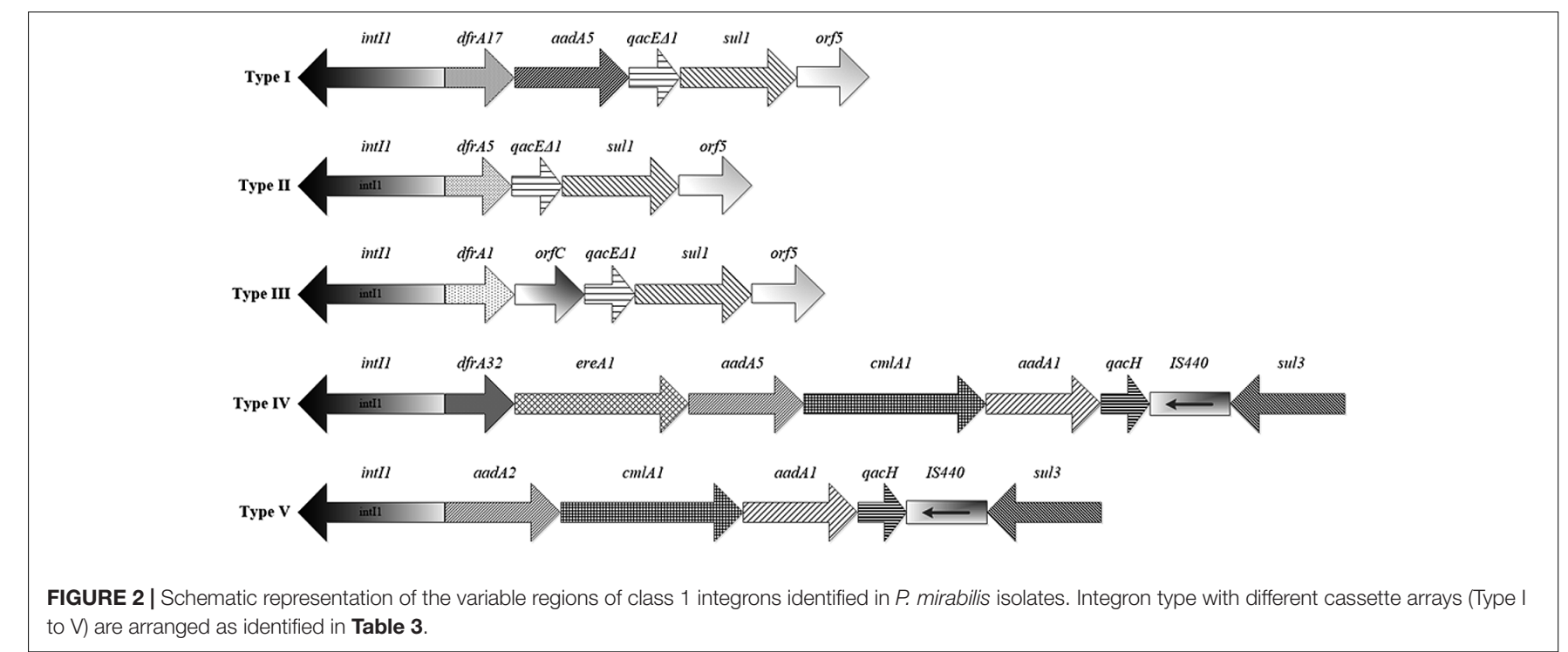


TABLE 3 | Characteristics of isolates carrying integrons.

\begin{tabular}{|c|c|c|c|c|c|}
\hline \multirow[t]{2}{*}{ Isolate } & \multicolumn{5}{|c|}{ Class 1 integrons } \\
\hline & Size (bp) & Gene cassette & Type $^{a}$ & qacE $\Delta 1-s u l 1$ & Classic or non-classic $^{b}$ \\
\hline PM1 & 1664 & dfrA17-aadA5 & 1 & + & Classic \\
\hline PM4 & 2900 & dfrA32-ereA-aadA2 & IV & - & Non-classic \\
\hline PM7 & 721 & dfra5 & $\|$ & + & Classic \\
\hline PM9 & 1664 & dfrA17-aadA5 & 1 & + & Classic \\
\hline PM13 & 1664 & dfrA17-aadA5 & 1 & + & Classic \\
\hline PM19 & 792 & aadA2 & V & - & Non-classic \\
\hline PM21 & 1163 & dfrA1-orfC & III & + & Classic \\
\hline PM24 & 721 & dfra5 & $\|$ & + & Classic \\
\hline PM35 & 1664 & dfrA17-aadA5 & 1 & + & Classic \\
\hline PM39 & 1664 & dfrA17-aadA5 & 1 & + & Classic \\
\hline
\end{tabular}

atype I-V represented five different integron structures in our study (Figure 2).

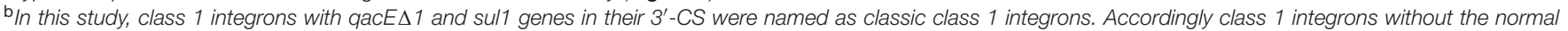
$3^{\prime}$-CS were named as non-classic class 1 integrons.

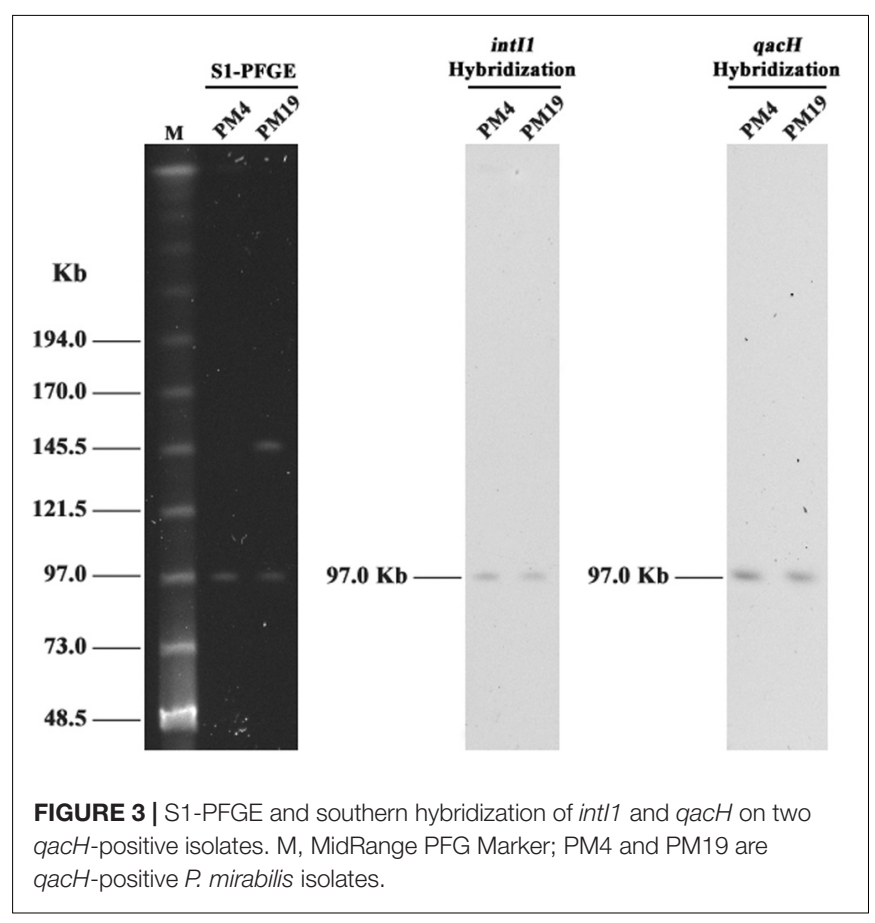

(Figure 3). PM4 contained a plasmid of $100 \mathrm{~kb}$ and PM19 contained two large plasmids of 100 and $150 \mathrm{~kb}$. Both specific probes for intI1 and qacH genes hybridized with the plasmids of $100 \mathrm{~kb}$ in the two isolates (Figure 3). Two transconjugants named PM4T and PM19T were obtained after conjugation experiments. Both of them exhibited resistance to streptomycin and chloramphenicol. However, increased MICs of BC were not observed in transconjugants (data not shown). PCR experiments confirmed that the transconjugants harbored the same gene structure of integrons as their donors (PM4T with $d f r A 32-$ ereA1-aadA2-cmlA1-aadA1-qacH-IS440-sul3 and PM19T with aadA2-cmlA1-aadA1-qacH-IS440-sul3). All the above indicated results confirmed that $q a c H$-associated class 1 integrons in the two studied isolates were located on conjugative plasmids of $100 \mathrm{~kb}$.

\section{DISCUSSION}

In the present study, fifty-two isolates of $P$. mirabilis were isolated from cooked meat samples. According to Wong et al. (2013), the rate of $P$. mirabilis in raw chicken carcass samples in Hong Kong was $86.2 \%$ (50/58), which was much higher than that $(29.2 \%)$ in our study. Cooking procedures kill most microorganisms that colonize raw meats, however, survival of microorganisms due to improper processing or cross-contamination of food after cooking may occur. This could be an explanation for the lower incidence of $P$. mirabilis in cooked meat products compared to raw meat samples.

Proteus mirabilis isolates in our study showed the MICs of BC ranging from 4 to $>32 \mu \mathrm{g} / \mathrm{mL}$, with MICs of $24 \mu \mathrm{g} / \mathrm{mL}$ most frequently. As there is no standard resistant breakpoint of $\mathrm{BC}$ for P. mirabilis, it is difficult to classify the isolates as BC susceptible, intermediate or resistance in this study. Although data on the BC susceptibility in $P$. mirabilis were scarce, several studies have reported the MICs of BC in Enterobacteriaceae isolates from different sources (Sidhu et al., 2002; Aarestrup and Hasman, 2004; Zhang et al., 2016). In the study of Zhang et al. (2016), E. coli isolated from retail meat showed the MICs of BC in the range of 16-64 $\mu \mathrm{g} / \mathrm{mL}$. Aarestrup and Hasman (2004) have shown that the MICs of BC for Salmonella $(n=156)$ and E. coli $(n=202)$ from food animals ranged from 64 to $256 \mu \mathrm{g} / \mathrm{mL}$ and from 16 to $128 \mu \mathrm{g} / \mathrm{mL}$, respectively. Compared with the studies mentioned above, $P$. mirabilis isolates in our study showed relatively lower MICs to BC. In another survey of Enterobacteriaceae from food, almost half of the isolates exhibited the MICs of BC with $<10 \mu \mathrm{g} / \mathrm{mL}$ (Sidhu et al., 2002). Actually, the user concentrations of BC in food industry are usually 200-1000 $\mu \mathrm{g} / \mathrm{mL}$ (Møretrø et al., 2017), which are much higher than the MICs of $P$. mirabilis in this study. In practical application, BC is commonly rinsed from surfaces (equipment, machines, floor etc.) with water after 
disinfection. This rinsing step, however, is not sufficient to remove all $\mathrm{BC}$ residues from surfaces and consequently, bacteria are likely to be exposed to diluted, sub-lethal BC concentrations (Buffet-Bataillon et al., 2012). Repeated exposure to sub-lethal $\mathrm{BC}$ concentrations may facilitate the development of resistance (Buffet-Bataillon et al., 2012). Therefore, it was not surprising that P. mirabilis isolates in the present study exhibited low-level of BC MICs.

In this study, the presence of QAC resistance genes was investigated. Our results showed that $m d f A$ and $y d g E / y d g F$ were the most prevalent among $P$. mirabilis, which was in agreement with the similar studies of E. coli (Zou et al., 2014; Zhang et al., 2016). Among the isolates tested in our study, qacE always occurred simultaneously with qacE $\Delta 1$. It was noted that the presence of $\operatorname{sugE}(p)$ was significantly associated with the higher MICs of BC $(P<0.05)$. Two qacH-positive isolates also exhibited relatively high MICs of $\mathrm{BC}(>32 \mu \mathrm{g} / \mathrm{mL})$. In previous research, the higher MICs of BC were associated with plasmid-encoded genes (Zou et al., 2014). Because each isolate harbored more than one QAC resistance gene, it is difficult to assess what level of BC resistance was contributed by each QAC resistance gene.

A previous study reported that aadA1 gene cassette was observed most commonly among the P. mirabilis isolates from retail meat products (Kim et al., 2005). In contrast, our results showed that $d f r A 17$-aadA5 was the most common cassette array, which is similar to other reports of foodborne $P$. mirabilis isolates in China (Shen et al., 2011). Interestingly, an uncommon integron gene cassette array $d f r A 32$-ereA1-aadA2 was found in one $P$. mirabilis isolate in this study. To the best of our knowledge, this is the first report of $d f r A 32$-ereA1-aadA2 in foodborne $P$. mirabilis. The integron gene cassette array $d$ frA32-ereA1aadA2 in our study showed $99.6 \%$ identity to that of Salmonella enterica (Krauland et al., 2010; GenBank accession number GU067642), 99.7\% identity to that of Laribacter hongkongensis (Feng et al., 2011; GenBank accession number GU726907) and 99.7\% identity to that of Aeromonas hydrophila (unpublished; GenBank accession number KJ543558). Recently, this cassette array was detected in clinical $P$. mirabilis isolates in Zhejiang Province of China (Wei et al., 2014), which had 99.6\% identity to our sequence. The high similarity of $d$ frA32-ereA1-aadA2 indicated that the class 1 integrons carrying this cassette array may be located on conjugative plasmids.

Classic class 1 integrons are composed of qacE $\Delta 1$ and sul1 genes in their $3^{\prime}$-CS. Recently, class 1 integrons without the typical $3^{\prime}$-CS have been found in some species of Enterobacteriaceae, including Escherichia coli, Salmonella, Shigella sonnei and Klebsiella pneumoniae (Antunes et al., 2007; Chang et al., 2009, 2011; Sáenz et al., 2010). In these previous studies, the $3^{\prime}$-CS of integrons was associated with qacH and sul3. $\mathrm{Xu}$ et al. (2009) reported that two clinical isolates of Pseudomonas aeruginosa carried a class 1 integron lacking $3^{\prime}$-CS, however, the genetic structure of these non-classic integrons was unclear. In our study, non-classic class 1 integrons lacking qacE $\Delta 1$ and sul1 genes were found in 2 of the 10 intI1-positive isolates (PM4 with dfrA32-ereA1-aadA2 and PM19 with aadA2). PCR amplifications revealed that the gene cassettes in the two isolates were followed by an unusual $3^{\prime}$-CS linked to qacH and sul3. The gene cassette organization dfrA32-ereA1-aadA2-cmlA1-aadA1qacH-IS440-sul3 and aadA2-cmlA1-aadA1-qacH-IS440-sul3 were identified in PM4 and PM19, respectively. Both of them contained cmlA1 coding for chloramphenicol resistance, aadA1 coding for streptomycin resistance, qacH coding for QAC resistance, insertion element IS440, and sul3 coding for sulfonamide resistance. It seems that sul3 had replaced sul1 in addition to the replacement of qacE $\Delta 1$ by $q a c H$ in the $3^{\prime}-\mathrm{CS}$ region. Although the gene structure $c m l A 1$-aadA1-qacH-IS440sul3 was commonly found in non-classic integrons (Antunes et al., 2007; Sáenz et al., 2010), the combination of dfrA32-ereA1aadA2-cmlA1-aadA1-qacH-IS440-sul3 was reported for the first time. This novel arrangement detected in P. mirabilis isolate from food has been included in GenBank with the accession number KY426918. A similar gene cassette array of qacHdfrA32-ereA1-aadA2-cmlA1-aadA1 was detected in Salmonella enterica serovar Stanley isolates from Taiwan (Krauland et al., 2010). Both of the two arrays carried the same gene cassettes, but the position of qacH was different. PM4 and PM19 had similar qacH-IS440-sul3-integron platforms with different gene cassette arrays, suggesting evolution of the genetic background by different recombinatorial events.

Our results demonstrated that non-classic class 1 integrons were located on conjugative plasmids of $100 \mathrm{~kb}$ in two tested isolates. Previous studies showed that the class 1 integrons lacking the normal $3^{\prime}$-CS and associated with qacH and sul3 genes were usually located on large plasmids of different size (between 70 and $240 \mathrm{~kb}$ ) and the conjugative plasmids of $100 \mathrm{~kb}$ were the most disseminated among the different isolates (Antunes et al., 2007; Sáenz et al., 2010). Pal et al. (2015) also found that plasmids with co-selection potential for resistance to disinfectants and antimicrobials tended to be large and conjugative. Conjugation experiments confirmed that the disinfectant and antimicrobial resistance genes of non-classic class 1 integrons in PM4 and PM19 were co-existed on the same conjugative plasmids and could be co-transferred to E. coli. When theses isolates are exposed to QACs, selection pressure from disinfectants could increase risks for the spread of QAC and antimicrobial resistance genes among the bacteria. Notably, two transconjugants containing $q a c H$ showed the same MICs of $\mathrm{BC}$ as the recipient in this study. Similar observations that the presence of QAC resistance genes didn't increase the MICs of $\mathrm{BC}$ in transconjugants has also been reported in a previous study (Zhang et al., 2016). There was the possibility that the agar dilution method used for susceptibility testing was not sensitive enough to detect the differences of BC MICs between the recipient and transconjugants.

\section{CONCLUSION}

Quaternary ammonium compounds resistance genes, including $m d f A, y d g E / y d g F, q a c E, q a c E \Delta 1, \operatorname{emrE}, \operatorname{sug} E(c)$, and $\operatorname{sugE}(p)$ were found in foodborne $P$. mirabilis isolates in this study. Our data demonstrated the presence of non-classic class 1 integrons with the gene structure qacH-IS440-sul3 among the isolates. Moreover, qacH-associated non-classic class 1 integrons were 
located on conjugative plasmids and therefore could constitute an effective way for co-dissemination of antimicrobial and disinfectant resistance genes.

\section{AUTHOR CONTRIBUTIONS}

TY, HW, and LS designed and supervised the study. XJ, LL, YL, and $\mathrm{KZ}$ performed the experiments. XJ analyzed data. XJ and TY drafted the manuscript.

\section{FUNDING}

This work was supported by the National Natural Science Foundation of China (31601568), the Key Project of Natural Science of the Education Department of Henan Province,

\section{REFERENCES}

Aarestrup, F. M., and Hasman, H. (2004). Susceptibility of different bacterial species isolated from food animals to copper sulphate, zinc chloride and antimicrobial substances used for disinfection. Vet. Microbiol. 100, 83-89. doi: 10.1016/j.vetmic.2004.01.013

Antunes, P., Machado, J., Peixe, L., and Mora, D. (2007). Dissemination of sul3containing elements linked to class 1 integrons with an unusual $3^{\prime}$ conserved sequence region among Salmonella isolates. Antimicrob. Agents Chemother. 51, 1545-1548. doi: 10.1128/AAC.01275-06

Bay, D. C., and Turner, R. J. (2009). Diversity and evolution of the small multidrug resistance protein family. BMC Evol. Biol. 9:140. doi: 10.1186/1471-2148-9-140

Buffet-Bataillon, S., Tattevin, P., Bonnaure-Mallet, M., and Jolivet-Gougeon, A. (2012). Emergence of resistance to antibacterial agents: the role of quaternary ammonium compounds-a critical review. Int. J. Antimicrob. Agents 39, 381-389. doi: 10.1016/j.ijantimicag.2012.01.011

Call, D. R., Singer, R. S., Meng, D., Broschat, S. L., Orfe, L. H., Anderson,

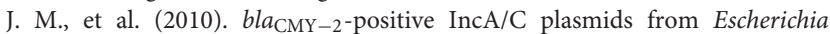
coli and Salmonella enterica are a distinct component of a larger lineage of plasmids. Antimicrob. Agents Chemother. 54, 590-596. doi: 10.1128/AAC. 00055-09

Ceccarelli, D., Salvia, A. M., Sami, J., Cappuccinelli, P., and Colombo, M. M. (2006). New cluster of plasmid-located class 1 integrons in Vibrio cholerae O1 and a $d f r A 15$ cassette-containing integron in Vibrio parahaemolyticus isolated in Angola. Antimicrob. Agents Chemother. 50, 2493-2499. doi: 10.1128/AAC. 01310-05

Chang, C., Fang, Y., Tsai, S., Chang, L., and Yu, W. (2009). Characterization of class 1 integrons and gene cassettes in clinical isolates of Klebsiella pneumoniae from Taiwan. Diagn. Microbiol. Infect. Dis. 65, 214-216. doi: 10.1016/j.diagmicrobio. 2009.06.005

Chang, C., Lu, P., Lin, C., Lee, T., Tsai, M., and Chang, L. (2011). Integron types, gene cassettes, antimicrobial resistance genes and plasmids of Shigella sonnei isolates from outbreaks and sporadic cases in Taiwan. J. Med. Microbiol. 60, 197-204. doi: 10.1099/jmm.0.022517-0

Chapman, J. S. (2003). Disinfectant resistance mechanisms, cross-resistance, and co-resistance. Int. Biodeterior. Biodegrad. 51, 271-276. doi: 10.1016/S09648305(03)00044- 1

Chu, Y. W., Afzal-Shah, M., Houang, E. T., Palepou, M. I., Lyon, D. J., Woodford, N., et al. (2001). IMP-4, a novel metallo-beta-lactamase from nosocomial Acinetobacter spp. collected in Hong Kong between 1994 and 1998. Antimicrob. Agents Chemother. 45, 710-714. doi: 10.1128/AAC.45.3.710-714. 2001

Clinical and Laboratory Standards Institute (2012). Performance Standards for Antimicrobial Susceptibility Testing: Twenty-Second Informational Supplement 2012, Vol. 32. Wayne, IL: CLSI.
China (15A180006 and 16A180011), the Key Scientific and Technological Project of Xinxiang (ZG15007) and the Doctoral Scientific Research Foundation of Henan Normal University.

\section{ACKNOWLEDGMENT}

The authors are grateful to Minggui Wang, Huashan Hospital, Fudan University, for kindly providing the E. coli J53 $\mathrm{Az}^{r}$.

\section{SUPPLEMENTARY MATERIAL}

The Supplementary Material for this article can be found online at: https://www.frontiersin.org/articles/10.3389/fmicb. 2017.02417/full\#supplementary-material

Cruz, C. D., and Fletcher, G. C. (2012). Assessing manufacturers' recommended concentrations of commercial sanitizers on inactivation of Listeria monocytogenes. Food Control 26, 194-199. doi: 10.1016/j.foodcont.2012. 01.041

Curiao, T., Cantón, R., Garcillán-Barcia, M. P., de la Cruz, F., Baquero, F., and Coque, T. M. (2011). Association of composite IS26-sul3 elements with highly transmissible IncI1 plasmids in extended-spectrum- $\beta$-lactamase-producing Escherichia coli clones from humans. Antimicrob. Agents Chemother. 55, 2451-2457. doi: 10.1128/AAC.01448-10

Farkas, A., Crăciunaş, C., Chiriac, C., Szekeres, E., Coman, C., and Butiuc-Keul, A. (2016). Exploring the role of coliform bacteria in class 1 integron carriage and biofilm formation during drinking water treatment. Microb. Ecol. 72, 773-782. doi: 10.1007/s00248-016-0758-0

Feng, J. L., Yan, H., Chowdhury, N., Neogi, S. B., Yamasaki, S., Shi, L., et al. (2011). Identification and characterization of integron-associated antibiotic resistant Laribacter hongkongensis isolated from aquatic products in China. Int. J. Food Microbiol. 144, 337-341. doi: 10.1016/j.ijfoodmicro.2010.10.014

Garza-Ramos, J. U., Sanchez-Martinez, G., Barajas, J. M., Suarez, S., SanchezPerez, A., Rojas-Moreno, T., et al. (2010). Variability of the bla $a_{\mathrm{IMP}-15^{-}}$ containing integrons, highly related to In95, on an endemic clone of Pseudomonas aeruginosa in Mexico. Microb. Drug Resist. 16, 191-195. doi: $10.1089 / \mathrm{mdr} .2010 .0017$

Garza-Ramos, U., Morfin-Otero, R., Sader, H. S., Jones, R. N., Hernández, E., Rodriguez-Noriega, E., et al. (2008). Metallo- $\beta$-lactamase gene $b l a_{\mathrm{IMP}-15}$ in a class 1 integron, In95, from Pseudomonas aeruginosa clinical isolates from a hospital in Mexico. Antimicrob. Agents Chemother. 52, 2943-2946. doi: 10.1128/ AAC.00679-07

Gillings, M. R. (2010). Biocide use, integrons and novel genetic elements. Microbiol. Aust. 31, 192-194. doi: 10.1038/ismej.2008.98

González-Rodríguez, M., Sanz, J., Santos, J., Otero, A., and García-López, M. (2002). Numbers and types of microorganisms in vacuum-packed coldsmoked freshwater fish at the retail level. Int. J. Food Microbiol. 77, 161-168. doi: 10.1016/S0168-1605(02)00048-X

Hegstad, K., Langsrud, S., Lunestad, B. T., Scheie, A. A., Sunde, M., and Yazdankhah, S. P. (2010). Does the wide use of quaternary ammonium compounds enhance the selection and spread of antimicrobial resistance and thus threaten our health. Microb. Drug Resist. 16, 91-104. doi: 10.1089/mdr. 2009.0120

Heir, E., Sundheim, G., and Holck, A. L. (1998). The Staphylococcus qacH gene product: a new member of the SMR family encoding multidrug resistance. FEMS Microbiol. Lett. 163, 49-56. doi: 10.1111/j.1574-6968.1998.tb13025.x

Jiang, X., Xu, Y., Li, Y., Zhang, K., Liu, L., Wang, H., et al. (2017). Characterization and horizontal transfer of $q a c H$-associated class 1 integrons in Escherichia coli isolated from retail meats. Int. J. Food Microbiol. 258, 12-17. doi: 10.1016/j. ijfoodmicro.2017.07.009 
Kazama, H., Hamashima, H., Sasatsu, M., and Arai, T. (1998). Distribution of the antiseptic-resistance gene $q a c E$ and $q a c E \Delta 1$ in Gram-negative bacteria. FEMS Microbiol. Lett. 159, 173-178.

Kim, S., Wei, C., and An, H. (2005). Molecular characterization of multidrugresistant Proteus mirabilis isolates from retail meat products. J. Food Prot. 68, 1408-1413. doi: 10.4315/0362-028X-68.7.1408

Koo, H. J., and Woo, G. J. (2011). Distribution and transferability of tetracycline resistance determinants in Escherichia coli isolated from meat and meat products. Int. J. Food Microbiol. 145, 407-413. doi: 10.1016/j.ijfoodmicro.2011. 01.003

Krauland, M., Harrison, L., Paterson, D., and Marsh, J. (2010). Novel integron gene cassette arrays identified in a global collection of multi-drug resistant non-typhoidal Salmonella enterica. Curr. Microbiol. 60, 217-223. doi: 10.1007/ s00284-009-9527-3

Li, D., Yang, M., Hu, J., Zhang, J., Liu, R., Gu, X., et al. (2009). Antibioticresistance profile in environmental bacteria isolated from penicillin production wastewater treatment plant and the receiving river. Environ. Microbiol. 11, 1506-1517. doi: 10.1111/j.1462-2920.2009.01878.x

Li, X., Shi, L., Yang, W., Li, L., and Yamasaki, S. (2006). New array of aacA4-catB3$d f r A 1$ gene cassettes and a noncoding cassette from a class-1-integron-positive clinical strain of Pseudomonas aeruginosa. Antimicrob. Agents Chemother. 50, 2278-2279. doi: 10.1128/AAC.01378-05

Meier, A. B., Guldimann, C., Markkula, A., Pöntinen, A., Korkeala, H., and Tasara, T. (2017). Comparative phenotypic and genotypic analysis of Swiss and Finnish Listeria monocytogenes isolates with respect to benzalkonium chloride resistance. Front. Microbiol. 8:397. doi: 10.3389/fmicb.2017.00397

Moreno, C., Romero, J., and Espejo, R. T. (2002). Polymorphism in repeated 16S rRNA genes is a common property of type strains and environmental isolates of the genus Vibrio. Microbiology 148, 1233-1239. doi: 10.1099/00221287-148-41233

Møretrø, T., Schirmer, B. C. T., Heir, E., Fagerlund, A., Hjemli, P., and Langsrud, S. (2017). Tolerance to quaternary ammonium compound disinfectants may enhance growth of Listeria monocytogenes in the food industry. Int. J. Food Microbiol. 241, 215-224. doi: 10.1016/j.ijfoodmicro.2016.10.025

Müller, A., Rychli, K., Muhterem-Uyar, M., Zaiser, A., Stessl, B., Guinane, C. M., et al. (2013). Tn6188-a novel transposon in Listeria monocytogenes responsible for tolerance to benzalkonium chloride. PLOS ONE 8:e76835. doi: 10.1371/ journal.pone.0076835

Naas, T., Mikami, Y., Imai, T., Poirel, L., and Nordmann, P. (2001). Characterization of In53, a class 1 plasmid- and composite transposon-located integron of Escherichia coli which carries an unusual array of gene cassettes. J. Bacteriol. 183, 235-249. doi: 10.1128/JB.183.1.235-249.2001

Pal, C., Bengtsson-Palme, J., Kristiansson, E., and Joakim Larsson, D. G. (2015). Co-occurrence of resistance genes to antibiotics, biocides and metals reveals novel insights into their co-selection potential. BMC Genomics 16:964. doi: 10.1186/s12864-015-2153-5

Partridge, S. R., Ginn, A. N., Paulsen, I. T., and Iredell, J. R. (2012). pEl1573 carrying blaIMP-4, from Sydney, Australia, is closely related to other IncL/M plasmids. Antimicrob. Agents Chemother. 56, 6029-6032. doi: 10.1128/AAC.01189-12

Sáenz, Y., Vinué, L., Ruiz, E., Somalo, S., Martínez, S., and Rojo-Bezares, B. (2010). Class 1 integrons lacking qacE $\Delta 1$ and sul1 genes in Escherichia coli isolates of food, animal and human origins. Vet. Microbiol. 144, 493-497. doi: 10.1016/j. vetmic.2010.01.026

Sandvang, D., Aarestrup, F. M., and Jensen, L. B. (1998). Characterization of integrons and antibiotic resistance genes in Danish multiresistant Salmonella enterica Typhimurium DT104. FEMS Microbiol. Lett. 160, 37-41. doi: 10.1111/ j.1574-6968.1998.tb12887.x
Shen, J., Yang, B., Xi, M., and Meng, J. (2011). Class I integron and antibiotic resistance of Salmonella and Proteus mirabilis in retail meat from Shaanxi Province. Food Sci. 32, 130-134.

Sidhu, M. S., Sørum, H., and Holck, A. (2002). Resistance to quaternary ammonium compounds in food-related bacteria. Microb. Drug Resist. 8, 393-399. doi: 10.1089/10766290260469679

Uzeh, R. E., Alade, F. A., and Bankole, M. (2009). The microbial quality of prepacked mixed vegetable salad in some retail outlets in Lagos, Nigeria. Afr. J. Food Sci. 3, 270-272.

Viedma, E., Juan, C., Acosta, J., Zamorano, L., Otero, J. R., Sanz, F., et al. (2009). Nosocomial spread of colistin-only-sensitive sequence type 235 Pseudomonas aeruginosa isolates producing the extend-spectrum $\beta$-lactamases GES- 1 and GES-5 in Spain. Antimicrob. Agents Chemother. 53, 4930-4933. doi: 10.1128/ AAC.00900-09

Wang, A., Yang, Y., Lu, Q., Wang, Y., Chen, Y., Deng, L., et al. (2008). Presence of $q n r$ gene in Escherichia coli and Klebsiella pneumoniae resistant to ciprofloxacin isolated from pediatric patients in China. BMC Infect. Dis. 8:68. doi: 10.1186/ 1471-2334-8-68

Wang, Y., Zhang, S., Yu, J., Zhang, H., Yuan, Z., Sun, Y., et al. (2010). An outbreak of Proteus mirabilis food poisoning associated with eating stewed pork balls in brown sauce, Beijing. Food Control 21, 302-305. doi: 10.1016/j.foodcont.2009. 06.009

Wei, Q., Hu, Q., Li, S., Lu, H., Chen, G., Shen, B., et al. (2014). A novel functional class 2 integron in clinical Proteus mirabilis isolates. J. Antimicrob. Chemother. 69, 973-976. doi: 10.1093/jac/dkt456

Wong, M. H. Y., Wan, H. Y., and Chen, S. (2013). Characterization of multidrug-resistant Proteus mirabilis isolated from chicken carcasses. Foodborne Pathog. Dis. 10, 177-181. doi: 10.1089/fpd.2012. 1303

Xu, Z., Li, L., Shirtliff, M. E., Alam, M. J., Yamasaki, S., and Shi, L. (2009). Occurrence and characteristics of class 1 and 2 integrons in Pseudomonas aeruginosa isolates from patients in southern China. J. Clin. Microbiol. 47, 230-234. doi: 10.1128/JCM.02027-08

Zhang, A., He, X., Meng, Y., Guo, L., Long, M., Yu, H., et al. (2016). Antibiotic and disinfectant resistance of Escherichia coli isolated from retail meats in Sichuan, China. Microb. Drug Resist. 22, 80-87. doi: 10.1089/mdr.2015. 0061

Zhao, S., Blickenstaff, K., Glenn, A., Ayers, S. L., Friedman, S. L., Abbott, J. W., et al. (2009). $\beta$-lactam resistance in Salmonella strains isolated from retail meats in the United States by the National antimicrobial resistance monitoring system between 2002 and 2006. Appl. Environ. Microbiol. 75, 7624-7630. doi: 10.1128/ AEM.01158-09

Zou, L., Meng, J., McDermott, P. F., Wang, F., Yang, Q., Cao, G., et al. (2014). Presence of disinfectant resistance genes in Escherichia coli isolated from retail meats in the USA. J. Antimicrob. Chemother. 69, 2644-2649. doi: 10.1093/jac/ dku197

Conflict of Interest Statement: The authors declare that the research was conducted in the absence of any commercial or financial relationships that could be construed as a potential conflict of interest.

Copyright (c) 2017 Jiang, Yu, Liu, Li, Zhang, Wang and Shi. This is an open-access article distributed under the terms of the Creative Commons Attribution License (CC BY). The use, distribution or reproduction in other forums is permitted, provided the original author(s) or licensor are credited and that the original publication in this journal is cited, in accordance with accepted academic practice. No use, distribution or reproduction is permitted which does not comply with these terms. 